\title{
Electroconvulsive therapy suppresses the neurotoxic branch of the kynurenine pathway in treatment-resistant depressed patients
}

Lilly Schwieler ${ }^{1 *}$, Martin Samuelsson 1,2, Mark A. Frye ${ }^{3}$, Maria Bhat ${ }^{4,5}$, Ina Schuppe-Koistinen 1,6, Oscar Jungholm", Anette G. Johansson ${ }^{5}$, Mikael Landén ${ }^{7,8}$, Carl M. Sellgren ${ }^{1,9,10}$ and Sophie Erhardt ${ }^{1}$

\begin{abstract}
Background: Neuroinflammation is increasingly recognized as contributing to the pathogenesis of depression. Key inflammatory markers as well as kynurenic acid (KYNA) and quinolinic acid (QUIN), both tryptophan metabolites, have been associated with depressive symptoms and suicidality. The aim of the present study is to investigate the peripheral concentration of cytokines and tryptophan and kynurenine metabolites in patients with unipolar treatment-resistant depression before and after electroconvulsive therapy (ECT), the most effective treatment for depression.

Methods: Cytokines in plasma from patients with major depressive disorder (MDD; $n=19)$ and healthy volunteers $(n=14)$ were analyzed with electrochemiluminescence detection. Tryptophan and kynurenine metabolites were detected with high-performance liquid chromatography (HPLC) and LC/MS. KYNA was analyzed in a second healthy control cohort $(n=22)$.

Results: Patients with MDD had increased plasma levels of interleukin (IL)-6 compared to healthy volunteers $(P<0.05)$. We also found an altered kynurenine metabolism in these patients displayed by decreased plasma levels of KYNA $(P<0.0001)$ as well as a significantly increased QUIN/KYNA ratio $(P<0.001)$. Plasma levels of tryptophan, kynurenine, and QUIN did not differ between patients and controls. Treatment with ECT was associated with a significant decrease in the plasma levels of tryptophan $(P<0.05)$, kynurenine $(P<0.01)$, and QUIN $(P<0.001)$, whereas plasma levels of KYNA did not change. The QUIN/KYNA ratio was found to significantly decrease in ECT-treated patients $(P<0.05)$. There was a significant inverse correlation between symptom severity and kynurenine levels at baseline $(r=-0.67, P=0.002)$.

Conclusions: This study confirms an imbalanced kynurenine pathway in MDD supporting the hypothesis of a netstimulation of $\mathrm{N}$-methyl-D-aspartic acid (NMDA) receptors in the disorder. Treatment with ECT profoundly decreased QUIN, an NMDA-receptor agonist previously suggested to be implicated in the pathogenesis of depression, an effect that might have bearing for the good clinical outcome of ECT.
\end{abstract}

Keywords: NMDA receptor, Cytokines, ECT, Quinolinic acid, Inflammation, IL-6, Kynurenic acid, Treatment-resistant depression

\footnotetext{
* Correspondence: lilly.schwieler@ki.se

'Department of Physiology and Pharmacology, Karolinska Institutet, SE-171

77 Stockholm, Sweden

Full list of author information is available at the end of the article
} 


\section{Background}

Major depressive disorder is a severe, life-threatening psychiatric illness, exposing the patient to direct risks, such as increased mortality due to cardiovascular disease, stroke, and alcohol abuse disorders. It is a leading cause of disability affecting millions of people worldwide and often causing chronic recurrent symptoms and profound socioeconomic burden [1]. With depression also follows a high risk of suicidal behavior, including attempts and completed suicides. More than 800,000 people die due to suicide every year, and it is estimated that for each adult who dies of suicide, there may have been more than 20 others attempting suicide [2]. Depression has been ranked as the fourth leading cause of disability worldwide by the World Health Organization [3], and the lifetime prevalence in high-income countries is $14 \%$ [ 4$]$.

The etiology of depression is still poorly understood, but recent hypotheses suggest that its development involves the activation of the innate immune system as well as alterations in glutamate excitotoxicity [5-7]. Indeed, increased concentrations of pro-inflammatory cytokines, chemokines, and acute-phase proteins have been observed in depressed patients. Increased circulating levels of interleukin (IL)-6 and C-reactive protein are the most frequently observed findings [8-10], but also, increased central levels of IL- 6 have been described $[11,12]$.

Although evidence of immune activation in depression is emerging, the functional relationship to aberrant brain neurotransmission and behavior still needs to be established. Recently, activation of the kynurenine pathway has been suggested as a specific pathogenic mechanism that can explain this link [13-16]. Tryptophan degradation along this pathway produces several neuroactive compounds, such as quinolinic acid (QUIN), an $N$-methylD-aspartic acid (NMDA)-receptor agonist, and kynurenic acid (KYNA), a blocker of the NMDA receptor and the alpha7* nicotinic receptor ( $\alpha 7 \mathrm{nAChR})$ [17]. Importantly, pro-inflammatory cytokines, especially interferon- $\gamma$ but also IL-1 $\beta$ and IL-6, are potent inducers of indoleamine 2,3 dioxygenase (IDO-1) and tryptophan 2,3-dioxygenase (TDO2), the two main enzymes regulating the first step of the kynurenine pathway [18-21]. We, and others, have previously shown that KYNA is elevated in the cerebrospinal fluid (CSF) and postmortem brain of patients with psychotic disorders, such as schizophrenia and bipolar disorder [22-28]. With regard to depression, excessive NMDA-receptor signaling, tentatively through an increased QUIN/KYNA ratio, has been suggested [14, 15]. Thus, in a recent study, we found that CSF levels of QUIN were elevated in suicide attempters, and approximately half of these patients suffered from major depression. CSF QUIN was also correlated to CSF IL-6, indicating that inflammation had triggered the synthesis of QUIN [14].
Enhanced QUIN density has also been observed in the anterior cingulate gyrus of suicide victims [29]. Studies of peripheral kynurenines in depression are not totally uniform, but similar to CSF studies, they point to an overweight of the toxic branch of the kynurenine pathway. Thus, decreased plasma levels of KYNA [30,31] and reduced plasma KYNA/QUIN and/or KYNA/3-hydroxykynurenine $(3-\mathrm{HK})$ ratios $[32,33]$ have been described in depressed patients.

Since the 1950s, more or less all medications used for depression target the monoaminergic system. The efficacy of these medications is limited, and a substantial proportion of patients experience residual symptoms and persistent functional impairments or do not even respond to treatment [34]. Typically, they also have a lag time of several weeks [35], which is not satisfactory for acute suicide ideation and severe depression. Therefore, there is a major need to develop more effective therapies to treat these disorders and/or their deleterious phases by exploring other therapeutic approaches. An alternative treatment for more severe major depression, treatmentresistant depression, or suicidality is electroconvulsive therapy (ECT). This therapy triggers a brief seizure, by applying electric currents that pass through the brain.

Despite the longstanding use of ECT, the exact neurobiological mechanisms underlying its efficacy are not yet understood. Studies have primarily focused on the changes in neurophysiology [36], neuroendocrine [37], and neurogenesis [38] after ECT treatment. However, only a limited number of studies have addressed functioning of the immune system in relation to ECT for depression.

The aims of the present study are to analyze cytokines and kynurenines in treatment-resistant patients with major depressive disorder (MDD) and healthy controls and to relate these biomarkers to depression scores. We also investigate whether ECT influences cytokines and kynurenines in these patients.

\section{Methods \\ Subjects \\ Patients}

Nineteen patients diagnosed with MDD (11 males and 8 females) and prescribed ECT by their treating psychiatrist were included in the study. Venipuncture failed in three patients at blood sampling after the third ECT administration, and one patient was excluded due to upper respiratory tract infection at the time of ECT treatment. Data from these patients are included only at baseline. The diagnosis was established by a psychiatrist using the complete clinical presentation and a Mini-International Neuropsychiatric Interview (MINI) [39] or by two psychiatrists using the Montgomery-Åsberg Depression Rating Scale (MADRS) [40]. MADRS was available for 18 patients at baseline and for 15 patients after three 
ECT. Patients under the age of 18 , involuntarily committed, subjected to ECT within 3 months prior to the study, or not able to understand the verbal and written information were excluded from the study. Eighteen patients had been adequately treated with oral antidepressant but had not responded, and one was treatment naïve. The concomitant drugs given were lithium $(n=1)$, antipsychotics $(n=3)$, benzodiazepines $(n=8)$, and hypnotic drugs $(n=12)$.

\section{Healthy volunteers and healthy controls}

Cohort 1 Fourteen male volunteers were mainly recruited among medical students, hospital staff members, and their relatives. They were all in good physical health and subjected to a medical check-up including laboratory tests (electrolytes, blood, thyroid, kidney, and liver) and a physical examination. All volunteers were free from medication for at least 1 month and free from any form of substance abuse, except for smoking (three healthy volunteers were smokers). Volunteers were subjected to a semi-structured interview using the Structured Clinical Interview for DSM-IV Disorders [41] or interviewed by a psychiatrist to exclude mental illness. None of them had a family history of major psychosis, major depression, or suicide in first- or second-degree relatives, and they were all found to be free from current signs of psychiatric morbidity or difficulties in social adjustment at the time of sampling.

Cohort 2 Population-based healthy controls were randomly selected by Statistics Sweden (SCB) and contacted by mail with a request for them to contact the study nurse if interested in participating in the study. Following a preliminary telephone screening, eligible persons were interviewed by experienced psychiatrists. Apart from psychiatric disorders and drug abuse, other excluded diagnoses were neurological conditions other than mild migraines, untreated endocrinological disorders, autoimmune disorders, pregnancy, and a family history of schizophrenia or bipolar disorder in firstdegree relatives. Additionally for this study, individuals who had inflammatory disorders were excluded. Twenty-two such healthy controls were selected to match the probands, based on sex and age.

\section{ECT and plasma sampling}

Patients were treated with ECT in the morning after at least $6 \mathrm{~h}$ of fasting at the University Hospital, Linköping, and at the Department of Clinical and Experimental Medicine, Division of Psychiatry, Faculty of Health Sciences, Linkoping University, Sweden. All patients received atropine before anesthesia was induced with thiopental. Muscle relaxation was induced with succinylcholine. The patients were ventilated with $100 \%$ oxygen. A Mecta spectrum 5000Q device was used to give right unilateral ECT treatments with ultrabrief or brief pulse waves, and dosage was adjusted by age, sex, and outcome. Electroencephalography and a stopwatch were used to monitor the seizures. All plasma samples were collected between 8 and 11 am and after a night of fasting.

\section{Policy and ethics}

This study was conducted in accordance with the latest Helsinki Protocol. The Ethics Committee of the Medical Faculty of Linköping University, Sweden; the Swedish Medical Products Agency; the Regional Ethics Committee in Stockholm; and the Swedish Data Inspection Board approved the study. All patients and healthy volunteers included received verbal and written information and gave their written informed consent. AstraZeneca did neither influence nor sponsor the clinical research performed at Linköping University or Karolinska Institutet. These data arise from a sample collection, not from a prospective trial, and is therefore not recorded in any clinical trial registry.

\section{Biochemical analyses}

Blood samples were collected in heparinized tubes from overnight-fasting healthy volunteers, healthy controls, and patients. In patients, blood samples were collected before the first ECT and after the third ECT. Blood samples were centrifuged at $(1438 \times g, 10 \mathrm{~min})$, and plasma was collected and stored at $-70{ }^{\circ} \mathrm{C}$ until analysis. All tryptophan metabolites were successfully detected in all plasma samples with an exception for KYNA and kynurenine not detected in one sample. In the second cohort of controls, only KYNA was analyzed.

\section{Analysis of KYNA}

Plasma samples were centrifuged at $21,000 \times g$, for $5 \mathrm{~min}$ after deproteination with an equal volume of perchloric acid (0.4 M, containing $0.1 \%$ sodium metabisulfite and $0.05 \%$ EDTA). The centrifugation procedure was repeated followed by an additional $70 \%$ perchloric acid (10 \% by volume). Analysis of KYNA was measured by high-performance liquid chromatography (HPLC) with fluorescence detection, and the method has been outlined in detail previously [26]. Briefly, $50 \mu \mathrm{L}$ of the deproteinized supernatant was injected to a ReproSilPur C18 reversed-phase column $(4 \times 150 \mathrm{~mm}$; Dr. Maisch $\mathrm{GmbH}$, Ammer-buch, Germany) using a fluorescence detector (Jasco Ltd., Hachioji City, Japan) with an excitation wavelength of $344 \mathrm{~nm}$ and emission wavelength of $398 \mathrm{~nm}$ (18-nm bandwith). A mobile phase containing $50 \mathrm{mM}$ sodium acetate $(\mathrm{pH}$ 6.2) and $7 \%$ acetonitrile was pumped through the reverse-phase column at a flow rate of $0.5 \mathrm{~mL} / \mathrm{min}$. Zinc acetate $(0.5 \mathrm{M})$ was delivered post-columnar by a Pharmacia P-500 
(GE Healthcare, Uppsala, Sweden) at a flow rate of $10 \mathrm{~mL} / \mathrm{h}$. The retention time of KYNA was approximately $7.5 \mathrm{~min}$ and was clear from other peaks with an exception for one sample that could not be distinguished due to an unknown peak at the same retention time. The sensitivity of the system was verified by analysis of a standard mixture of KYNA with concentrations from 0.25 to $30 \mathrm{nM}$, which resulted in a linear standard plot. The lower detection limit of the system was set to $0.625 \mathrm{nM}$. Some samples were analyzed in duplicates and confirmed that the intraindividual variation did not exceed $5 \%$.

\section{Analysis of tryptophan, kynurenine, and QUIN}

Plasma and standard samples $(100 \mu \mathrm{L})$ were diluted two times with internal standard solution in $5 \%$ formic acid and filtered at $2500 \times g$ for $60 \mathrm{~min}$ at $10{ }^{\circ} \mathrm{C}$ using $10-\mathrm{kDa}$ Ultrace ${ }^{\circledR}-10$ filter plates from Millipore. Internal Standards (IS) were added to each standard and plasma sample. Metabolites were purchased from Sigma-Aldrich (QUIN, kynurenine, and ${ }^{2} \mathrm{H}_{5}$-tryptophan) and Synfine Research Inc., Ontario, Canada ( ${ }^{13} \mathrm{C}_{3}^{15} \mathrm{~N}_{1}$-QUIN).

After centrifugation, $7.5 \mu \mathrm{L}$ of the filtrate was injected using a Waters Acquity HPLC system equipped with a SymmetryShield $^{\mathrm{Tm}}$ RP18 $2.1 \times 100 \mathrm{~mm}, 3.5-\mu \mathrm{m}$ particle column. The detection was performed using a Waters Xevo TQ triple quadrupole mass spectrometer operating in positive ionization MS/MS configuration. The mobile phase was run at a flow rate of $300 \mu \mathrm{L} / \mathrm{min}$ and consisted of $2.1 \%$ formic acid in MilliQ water (A phase) and acetonitrile (B phase) starting with $5 \%$ acetonitrile for 2 min following gradient elution, total run time of $10 \mathrm{~min}$. The formic acid and acetonitrile were purchased as MS-grade from Sigma-Aldrich. The mass spectrometer was tuned for each analyte and set at a capillary voltage of $3.0 \mathrm{~V}$, cone voltage of $16 \mathrm{~V}$, source temperature of $150{ }^{\circ} \mathrm{C}$, desolvation temperature of $350{ }^{\circ} \mathrm{C}$, and desolvation gas flow of $650 \mathrm{~L} / \mathrm{h}$. Mass spectral transition for QUIN was $\mathrm{m} / \mathrm{z} 168>106$, kynurenine $209>146$, and tryptophan $205>118$ using collision energies of 14,18 , and $24 \mathrm{eV}$, respectively. Retention times for QUIN, kynurenine, and tryptophan were $1.2,1.7$, and $3.6 \mathrm{~min}$, respectively.

\section{Cytokine analyses}

IL-1 $\beta$, IL-2, IL-6, IL-8, IL-10, IL-12p70, tumor necrosis factor (TNF)- $\alpha$, interferon (IFN)- $\gamma$, and granulocytemacrophage colony-stimulating factor (GM-CSF) were quantified in plasma using a customized Human UltraSensitive 9-Plex Kit (MesoScale Discovery, Gaithersburg, MD, USA) in 2013. The assays were analyzed as per the manufacturers' protocol (http://www.mesoscale.com). Intra-assay coefficient of variation was below $25 \%$ for all cytokines presented. The limits of detection (LOD) were
IL-1 $\beta$ (0.36 pg/mL), IL-2 (0.35 pg/mL), IL-6 (0.27 pg/mL), IL-8 (0.09 pg/mL), IL-10 (0.21 pg/mL), IL-12p70 (1.4 pg/ $\mathrm{mL})$, TNF- $\alpha,(0.5 \mathrm{pg} / \mathrm{mL}), \mathrm{IFN}-\gamma(0.74 \mathrm{pg} / \mathrm{mL})$, and GMCSF $(0.20 \mathrm{pg} / \mathrm{mL})$.

\section{Statistical analysis}

All values are given as median and with interquartile range (IQR). Nonparametric tests were used due to a small sample size in each group (D'Agostino \& Pearson omnibus normality test). Differences regarding plasma levels of cytokines and kynurenine metabolites between healthy controls and patients with depression were established using Mann-Whitney $U$ test. Wilcoxon matched-pairs signed-rank test was used when computing differences in MADRS scores, plasma concentrations of cytokines, and kynurenine metabolites in patients before and after treatment with ECT. All correlation analyses were performed using a Spearman rank correlation analysis. Significance was assumed for all values where $P<0.05$. All reported $P$ values are two sided, and all analyses were corrected for age and gender when appropriate. The analyses were done using SPSS $^{\circledR}$ Statistics 20.0 (IBM Inc., Chicago, IL, USA) or Prism 6.0 (GraphPad Software Inc., La Jolla, CA).

\section{Results \\ Demographics}

In the present study, 19 patients (11 males and 8 females), 14 (all males) healthy volunteers, and 22 healthy controls (13 males and 9 females) were included. The median age was significantly higher in patients (41.0 years, IQR 25.054.0 years) than in healthy volunteers (24.0 years, IQR 23.0-26 years, $P<0.004$, Mann-Whitney $U$ test) but similar to the healthy controls (40.5 years, IQR 34.8-60.5 years). Patients were clinically evaluated with MADRS before ECT, and the median MADRS score for these patients was 37.0 (IQR 28.8-44.3). Following three ECT administrations, MADRS significantly decreased to 20.5 (IQR 14.026.5, $P<0.001)$.

\section{Cytokines in patients with MDD and healthy male volunteers}

Plasma levels of IL-6, IL-8, IL-10, and TNF- $\alpha$ were detected in 13 of the 14 healthy male volunteers and in all 19 unipolar depressed patients with an exception for IL10 and IL- 6 that was not detected in one patient with MDD. The other cytokines analyzed were undetectable (IL-2) or found to be very close to the detection limit of the assay. Thus, IL- $1 \beta$, IFN- $\gamma$, GM-CSF, and IL-12p70 were only detected in a limited number of samples and therefore not further analyzed. Plasma IL-6 concentration was elevated in patients with MDD $(1.46 \mathrm{pg} / \mathrm{mL}$, IQR $0.80-4.0 \mathrm{pg} / \mathrm{mL}, n=18$ ) compared to healthy male volunteers $(0.85 \mathrm{pg} / \mathrm{mL}$, IQR $0.72-1.25 \mathrm{pg} / \mathrm{mL}, n=13$; 
$P=0.029$, Fig. 1a). When stratifying the analysis by sex, IL-6 was still significantly increased in male patients with MDD ([n=11] $1.46 \mathrm{pg} / \mathrm{mL}$, IQR $1.17-4.68 \mathrm{pg} / \mathrm{mL}$ vs. the healthy male volunteers $[n=13] 0.85 \mathrm{pg} / \mathrm{mL}$, IQR 0.72 $1.25 \mathrm{pg} / \mathrm{mL} ; P=0.0029)$. No correlation between age and IL-6 was observed in either patients $(\rho=-0.45 ; P=0.86)$ or in healthy male volunteers $(\rho=-0.14 ; P=0.63)$. We could not find any differences in the plasma levels of the other cytokines detected (IL-8, IL-10, and TNF- $\alpha$ ) between patients with MDD and healthy male volunteers (Fig. 1b-d).

Tryptophan and kynurenines in patients with MDD, healthy male volunteers, and healthy controls

Plasma concentrations of KYNA were markedly decreased in patients with MDD (23.8 nM, IQR 17.6$31.8 \mathrm{nmol} / \mathrm{L}, \quad n=18)$ compared to KYNA levels in healthy male volunteers $(42.4 \mathrm{nmol} / \mathrm{L}$, IQR $31.8-$ $51.2 \mathrm{nmol} / \mathrm{L}, n=14 ; P<0.0001, P$ [adjusted for age] = 0.001 , Fig. 2d). An increased QUIN/KYNA ratio in MDD (22.9, IQR 19.6-40.1, $n=18)$ compared to healthy volunteers was also observed (14.4, IQR 11.8-19.4, $n=$ 14 ; $P<0.001, P$ [adjusted for age] $=0.013$, Fig. 2 f). In patients and in healthy controls, we did not observe an effect of sex on either KYNA levels $(P=0.48$ and $P=$ 0.66 , respectively) or the QUIN/KYNA ratio $(P=0.38$, only patients).

No differences in the plasma levels of tryptophan or any of the other kynurenine metabolites (kynurenine, QUIN, or the ratio of kynurenine/tryptophan) were detected between patients with MDD and healthy volunteers (Fig. 2). Since the first control cohort included only male volunteers and were significantly younger than patients, plasma KYNA was measured in an age- and gender-matched second cohort of controls. Plasma KYNA was also here found to be significantly lower in patients with MDD. Plasma KYNA levels in the second cohort of controls were $40.6 \mathrm{nmol} / \mathrm{L}$, IQR 33.0 $54.8 \mathrm{nmol} / \mathrm{L}(n=22 ; P<0.0001)$.

\section{Tryptophan metabolites and cytokines after treatment with ECT in patients with MDD}

Analysis of plasma tryptophan and kynurenine metabolites after treatment with ECT in patients with MDD revealed that plasma KYNA levels did not change but that plasma levels of tryptophan, kynurenine, and QUIN significantly decreased after three ECT (Fig. 3a, b, and e.)
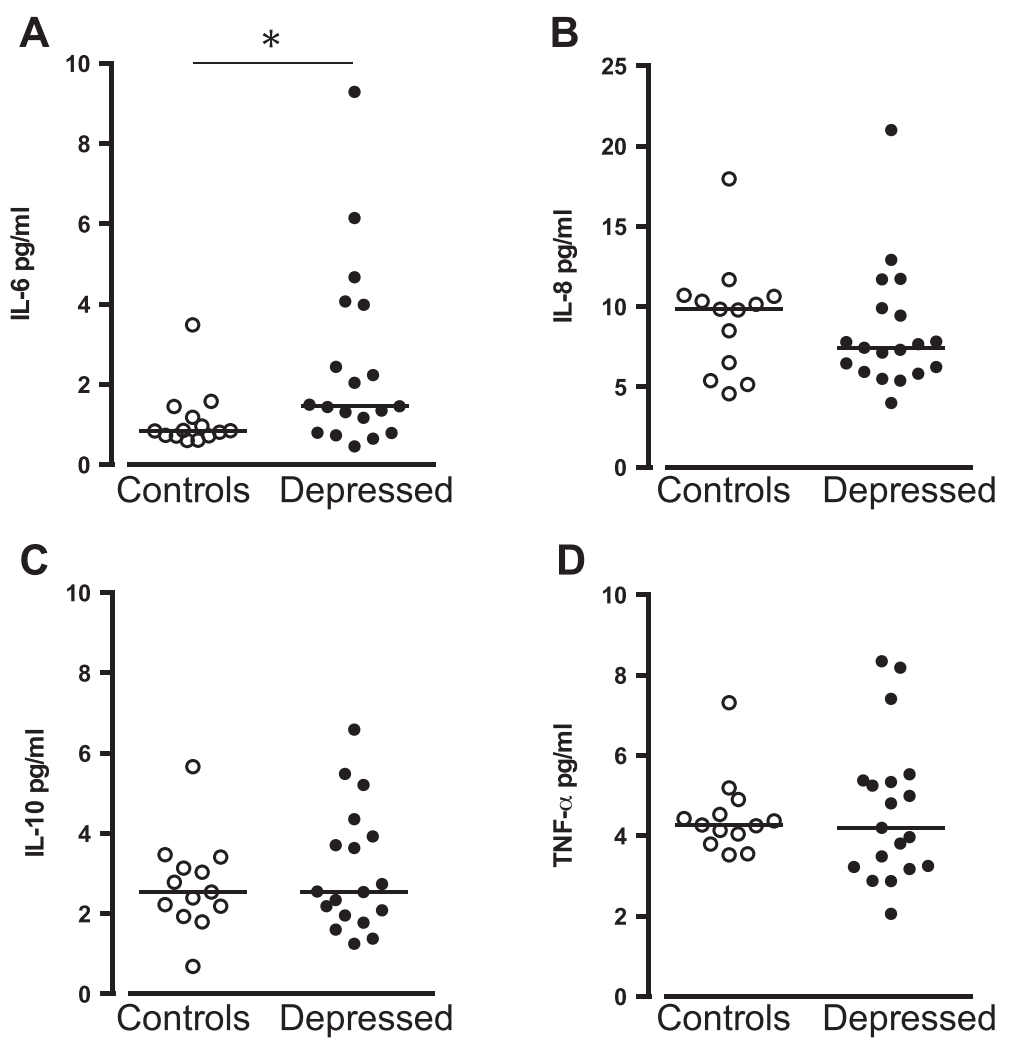

Fig. $1 \mathrm{IL}-6$ (a), IL-8 (b), IL-10 (c), and tumor necrosis factor-a (TNF-a) (d) in the plasma of patients with MDD $(n=18-19)$ and controls $(n=13)$. Each point represents the concentration of a single plasma sample and the horizontal lines the median for each group. ${ }^{*} P<0.05$ (Mann-Whitney $U$ test, reported $P$ value are two sided) 


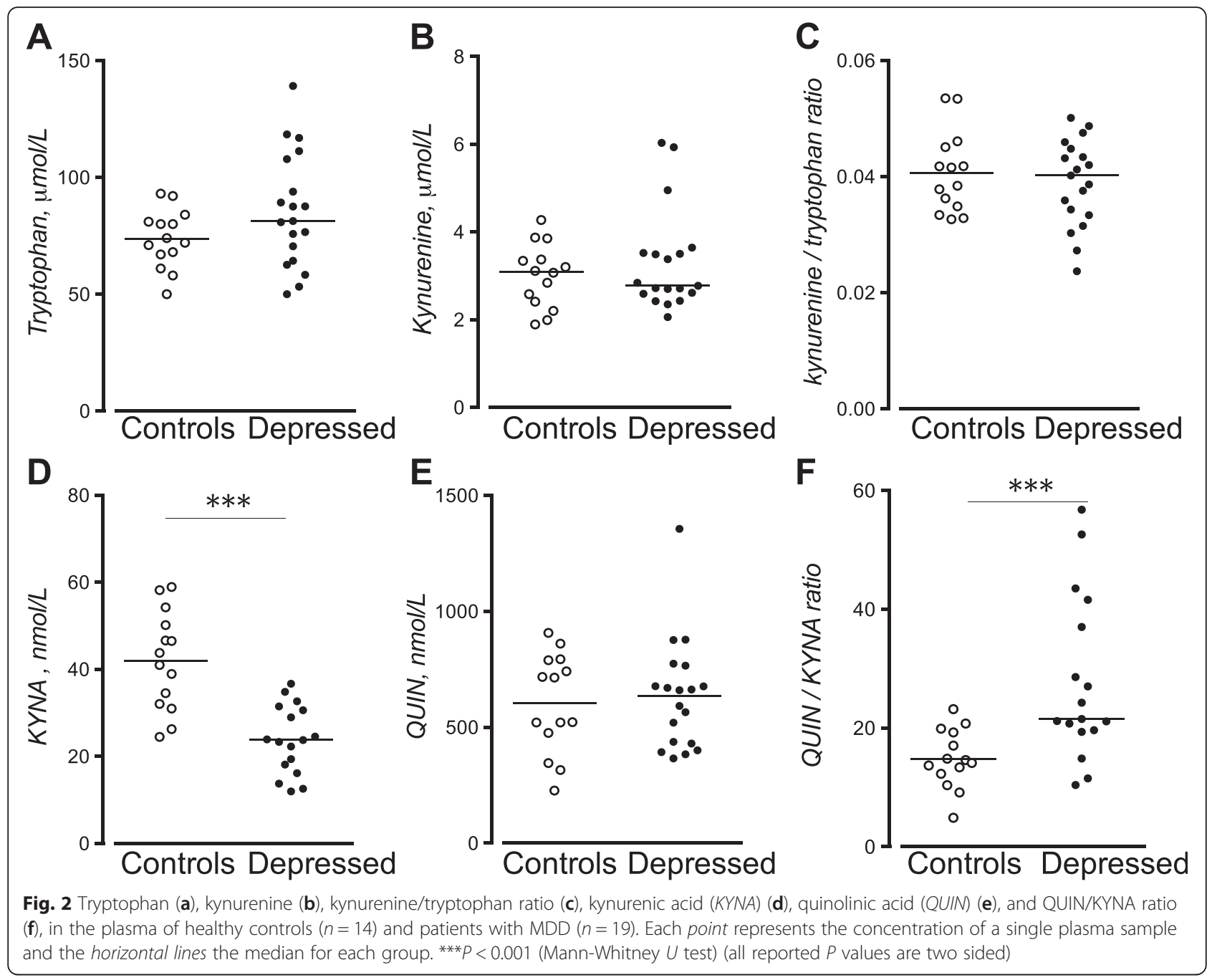

The QUIN/KYNA ratio significantly decreased after three ECT treatments $(P<0.05$, see Fig. $3 f)$.

The most pronounced effect of ECT was observed with regard to plasma QUIN levels, which was found to decrease in $80 \%$ (12 out of 15) of the patients. No changes in cytokine levels were found after three ECT treatments (data not shown).

\section{Correlation between kynurenine and MADRS score in patients with MDD}

Plasma kynurenine was found to negatively correlate to MADRS score in patients with MDD before treatment with ECT $(\rho=-0.67 ; P=0.002$, Fig. 4a). This correlation was not observed after three ECT administrations $(\rho=-0.13$; $P=0.66$, Fig. 4b). Neither did tryptophan nor any other kynurenine metabolite or cytokine correlate to the MADRS score before or after ECT. We could not find any association between changes in the concentrations of tryptophan metabolites and the antidepressive response (data not shown).

\section{Discussion}

In the present study, we investigated the peripheral concentration of cytokines and kynurenine metabolites in unipolar patients diagnosed with an ongoing severe major depressive episode. Our data, showing increased plasma levels of IL-6, decreased KYNA levels, and an increased QUIN/KYNA ratio in these patients, supports the view of an ongoing low-grade inflammation [42] and an imbalanced kynurenine pathway [30-33] in MDD. The finding that the QUIN/KYNA ratio was increased in patients supports the hypothesis of a netstimulation of NMDA receptors in depression [30-33]. The observation of low plasma KYNA was confirmed in a second, age- and gender-matched, healthy control cohort. Furthermore, we found that treatment with ECT induced major changes in peripheral kynurenine metabolites in patients with MDD. Thus, plasma levels of tryptophan, kynurenine, and QUIN significantly decreased after a series of three ECT administrations whereas plasma levels of KYNA did not change. Interestingly, following 


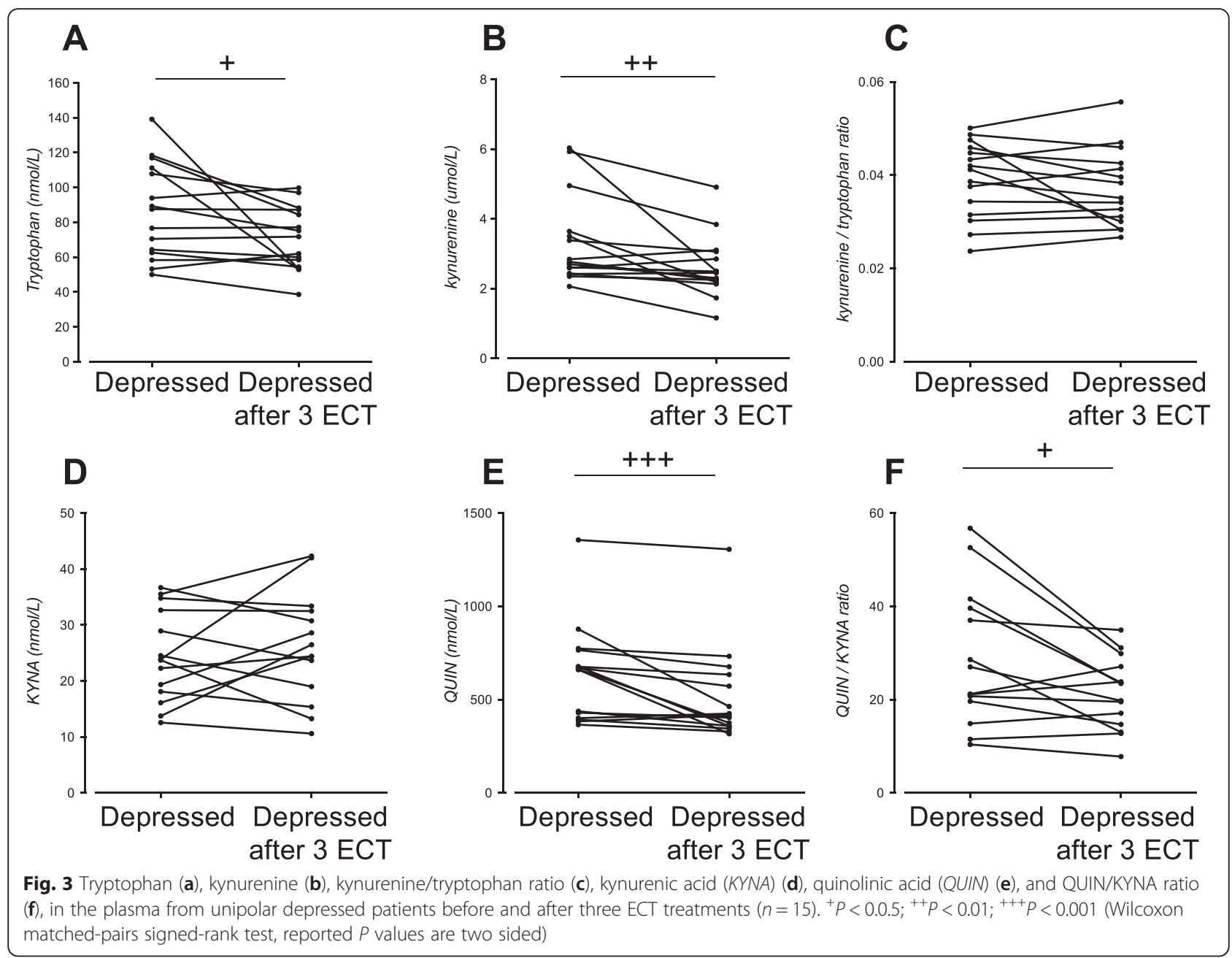

three ECT, plasma QUIN levels were found decreased in $80 \%$ of all patients. In addition, ECT was associated with a reduction in the QUIN/KYNA ratio, suggesting that NMDA receptors are to a lesser extent stimulated. In line with these findings, reduction in both CSF QUIN levels and in the CSF QUIN/KYNA ratio has previously been observed in suicide attempters following improvement of their depressive symptoms [14].

In the present study, we found increased plasma levels of IL-6 in patients with MDD. These results are in line

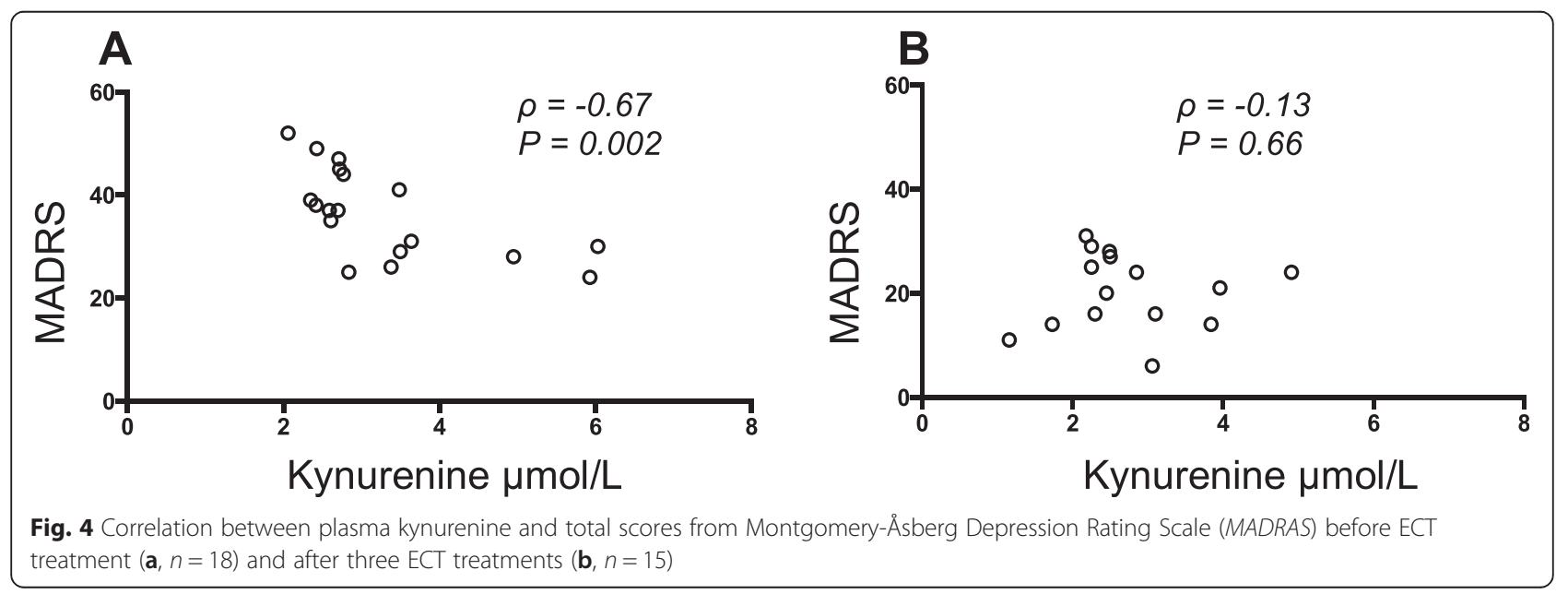


with other studies showing increased levels of IL-6 in patients with depression $[8,12,43]$. It is well known that pro-inflammatory cytokines, including IL-6, can induce IDO, the rate-limiting enzyme of the kynurenine pathway. Thus, injection of IL-6 into rat hippocampus is associated with the induction of IDO [19] and fetal human cortical astrocytes respond to application of IL-6 with an increased KYNA synthesis [20]. In line with this, we recently found significantly positive correlation between IL-6 and kynurenine metabolites in CSF, both in suicidal attempters [14] as well as in patients with schizophrenia [20], suggesting an intimate interplay between this cytokine and the kynurenine pathway in the central nervous system. However, in the present study, such a correlation was not observed, indicating that this mechanism might be brain specific.

In line with previous studies, we also here found low levels of plasma KYNA in patients with MDD. Since recent studies show that age and gender influence plasma KYNA levels [44, 45], we included a second cohort of age- and gender-matched healthy controls. Also compared to this group, plasma KYNA was significantly decreased in patients with MDD. A challenging question is why peripheral KYNA is decreased in depressed patients. It has been suggested that a genetic variant in one of the enzymes catalyzing the final step in the synthesis of KYNA, kynurenine aminotransferase (KAT) III, is responsible [46]. Reduced expression or activity of the KAT III enzyme might thus lead to diminished synthesis of KYNA and consequently an imbalance between KYNA and QUIN $[14,15,32,33,46]$. It was recently reported that the total hippocampal and amygdala volume in patients with MDD correlated with a reduced plasma KYNA/QUIN ratio, indicating that an overweigh of the toxic branch of the kynurenine pathway may be of importance for the neurodegeneration occurring in these patients [33]. The ability of ECT to significantly decrease QUIN levels could thus be of clinical importance. Indeed, a recent longitudinal ECT study shows that repeated ECT administration increases the levels of KYNA, shifting the balance toward the neuroprotective branch of the kynurenine pathway. Thus, serum levels of KYNA and KYNA/3-HK increased over time with the most pronounced effect on KYNA after 3 months [47]. In the present study, we did not observe any increase in the plasma levels of KYNA, but since our study only lasted for 2 weeks, longitudinal changes cannot be excluded. Conceivably, the mechanism of action of ECT on patients with MDD could be due to its ability to abate NMDA-receptor stimulation by decreasing the levels of QUIN and/or strengthen NMDA-receptor antagonism by increasing the levels of KYNA. Interestingly, ketamine, an NMDA-receptor antagonist, has also been reported to exert a rapid antidepressant effect in patients with depression [48-50]. This effect of ketamine further suggests that depression could be directly associated with deficits in glutamatergic synaptic transmission.

In the present study, we also found a slight decrease of plasma tryptophan after repeated ECT treatments in line with a previous study [51]. Earlier studies, however, provide an inconsistent picture in this regard. Thus, unaltered peripheral levels of tryptophan have been reported in depressed patients after single or repeated ECT administrations [47, 52, 53], whereas two other studies showed increased plasma tryptophan levels after ECT [54, 55]. The reason for the variability between studies is not obvious but could be due to differences in tryptophan measured (free or total), number of ECT treatments, or time of sampling after the last ECT. It has also been suggested that anesthesia used during ECT could affect the plasma levels of tryptophan [53].

In line with a recent study [46], a negative correlation between plasma kynurenine and depression scores was observed. This might indicate that high levels of peripheral kynurenine are protective against depressive symptoms. Indeed, kynurenine can be effectively transported over the blood-brain barrier (BBB) through the neutral amino acid transporter. Approximately $60 \%$ of brain kynurenine is coming from peripheral sources, and in the brain, kynurenine is transaminated to KYNA by the KAT enzymes in astrocytes or to QUIN by kynurenine 3-monooxygenase (KMO) in microglial cells. Compared to the higher capacity of KAT enzymes, displaying $K_{\mathrm{m}}$ values in the low milli-molar range [56], the KMO enzyme gets saturated at relatively low concentrations $\left(K_{\mathrm{m}} \approx 20 \mu \mathrm{M}\right)$ [57] and can therefore act as a ratelimiting step in the synthesis of QUIN. High levels of peripheral sources of kynurenine might, in this regard, rather contribute to increased levels of brain KYNA, which will guard against excessive NMDA-receptor stimulation and increase the threshold for depressive symptoms. In line with this hypothesis, we recently found that CSF KYNA was negatively associated with MADRS scores. Thus, the lower CSF KYNA, the worse degree of depression and suicidal symptoms [15]. In healthy volunteers, we recently detected a positive correlation between plasma kynurenine and CSF KYNA (unpublished observations), supporting the theory that the plasma levels of kynurenine observed in the present study reflect the central levels of KYNA in the patients.

In the present study, we observed that changes in the plasma QUIN levels associated with ECT treatment but not to the response of ECT as measured with MADRS. This is in line with a earlier study in depressed patients were CSF QUIN levels were found to correlate only to total scores on the Suicide Intent Scale but not to the mean score of MADRS or to the MADRS suicidality item [14]. This may indicate that QUIN does not affect 
depressive symptoms in general but rather has specific effects on suicidal behavior. This may also explain why we did not find any association between changes in the concentrations of QUIN after ECT and the antidepressive response.

The results of the present study should be interpreted in light of its limitations. Firstly, we only had excess to plasma and not CSF, restricting our analysis to peripheral markers. Cytokines as well as the kynurenine metabolites, KYNA and QUIN, do not easily pass the BBB, and therefore, plasma levels of cytokines and kynurenines may not reflect changes in the brain. Secondly, for ethical reasons, all patients were on different drug treatments. Statistical corrections for drug treatment were not eligible in this small cohort of patients, and therefore, we cannot fully rule out that the pharmacological treatment did not influence contractions of cytokines or kynurenines.

\section{Conclusions}

In conclusion, this study shows that ECT treatment reduces plasma QUIN levels in $80 \%$ of the patients and that the treatment was associated with a reduction in the QUIN/KYNA ratio. Furthermore, we confirm previous studies showing decreased peripheral KYNA levels and an increased QUIN/KYNA ratio in patients, suggestive of a netstimulation of NMDA receptors in MDD. Our findings strengthen the hypothesis that kynurenine metabolites are key players in the pathophysiology of MDD and the capacity of ECT to decrease the neurotoxic branch of the kynurenine pathway might therefore be of clinical relevance.

\footnotetext{
Abbreviations

3-HK: 3-hydroxykynurenine; BBB: blood-brain barrier; CSF: cerebrospinal fluid; ECT: electroconvulsive therapy; GM-CSF: granulocyte-macrophage colonystimulating factor; IDO-1: indoleamine 2,3 dioxygenase; IFN- $\gamma$ : interferon- $\gamma$; IL-6: interleukin-6; IQR: interquartile range; KAT: kynurenine aminotransferase; KMO: kynurenine 3-monooxygenase; KYNA: kynurenic acid;

MADRS: Montgomery-Åsberg Depression Rating Scale; MDD: major depressive disorder; NMDA: N-methyl-D-aspartic acid; QUIN: quinolinic acid; TDO2: tryptophan 2,3-dioxygenase; TNF-a: tumor necrosis factor-a; a7nAChR: alpha7* nicotinic receptor.
}

\section{Competing interests}

The authors declare that they have no competing interests.

\section{Authors' contributions}

LS, MS, and SE designed the study. LS and SE drafted the report. CS and LS were responsible for the statistical analyses. MS collected all the clinical data. LS performed the cytokine analysis. MB, IS-K, and OJ were responsible for the mass-spect and HPLC analysis. All authors contributed to the interpretation of the results, provided critical revision of the report, and approved the final manuscript. All authors have also ensured that questions related to the accuracy or integrity of any part of the work are appropriately investigated and resolved.

\section{Acknowledgements}

This work was supported by grants from the Swedish Medical Research Council (2009-7053; 2013-2838), the Swedish Brain Foundation, Svenska Läkaresällskapet, Petrus och Augusta Hedlunds Stiftelse, Östergötland County Council, the AstraZeneca-Karolinska Institutet Joint Research Program in
Translational Science, and the Karolinska Institutet (KID). The collection of population-based controls was funded by the Swedish Medical Research Council (K2014-62X-14647-12-51) and the Swedish Federal Government under the LUA/ALF agreement (ALF 20130032). We thank the patients, healthy volunteers, and healthy controls for their participation and express our gratitude toward the health professionals who facilitated our work; in particular, we would like to thank the research nurses. There are no commercial associations that might pose a conflict of interest in connection with the manuscript. The clinical research study was sponsored by Linköping University Hospital and Karolinska Institutet with an AstraZeneca in-kind contribution to the sample analysis for the present paper.

\section{Author details}

${ }^{1}$ Department of Physiology and Pharmacology, Karolinska Institutet, SE-171 77 Stockholm, Sweden. ${ }^{2}$ Department of Clinical and Experimental Medicine, Section of Psychiatry, Faculty of Health Sciences, Linköping University, Linköping, Sweden. ${ }^{3}$ Department of Psychiatry \& Psychology, Mayo Clinic College of Medicine, Rochester, MN, USA. ${ }^{4}$ Protein Biomarkers, Personalized Healthcare \& Biomarker Laboratories, Innovative Medicines, AstraZeneca, Gothenburg, Sweden. ${ }^{5}$ Department of Clinical Neuroscience, Karolinska Institutet, Stockholm, Sweden. ${ }^{6}$ Science for Life Laboratory, Proteomics \& Nanobiotechn, Karolinska Institutet, Stockholm, Sweden. Institute of Neuroscience and Physiology, The Sahlgrenska Academy at Gothenburg University, Gothenburg, Sweden. ${ }^{8}$ Department of Medical Epidemiology and Biostatistics, Karolinska Institutet, Stockholm, Sweden. ${ }^{9}$ Stanley Center for Psychiatric Research, Broad Institute of MIT and Harvard, Cambridge, MA, USA. ${ }^{10}$ Department of Psychiatry, Massachusetts General Hospital, Harvard Medical School, Boston, MA, USA.

Received: 22 December 2015 Accepted: 21 February 2016

Published online: 29 February 2016

\section{References}

1. Kessler RC, Berglund P, Demler O, Jin R, Koretz D, Merikangas KR, et al. The epidemiology of major depressive disorder: results from the National Comorbidity Survey Replication (NCS-R) National Comorbidity Survey Replication. JAMA. 2003;289(23):3095-105.

2. WHO report (2014), Preventing suicide: a global imperative. http://apps.who. int/iris/bitstream/10665/131056/1/9789241564779_eng.pdf.

3. Murray CJ, Lopez AD. Measuring the global burden of disease. N Engl J Med. 2013;369(5):448-57.

4. Kessler RC, Bromet EJ. The epidemiology of depression across cultures. Annu Rev Public Health. 2013;3(34):119-38.

5. Dantzer R, O'Connor JC, Freund GG, Johnson RW, Kelley KW. From inflammation to sickness and depression: when the immune system subjugates the brain. Nat Rev Neurosci. 2008;9(1):46-56.

6. Leonard B, Maes M. Mechanistic explanations how cell-mediated immune activation, inflammation and oxidative and nitrosative stress pathways and their sequels and concomitants play a role in the pathophysiology of unipolar depression. Neurosci Biobehav Rev. 2012;36(2):764-85.

7. Miller $\mathrm{AH}$, Haroon E, Raison $\mathrm{CL}$, Felger JC. Cytokine targets in the brain: impact on neurotransmitters and neurocircuits. Depress Anxiety. 2013:30(4):297-306.

8. Dowlati Y, Herrmann N, Swardfager W, Liu H, Sham L, Reim EK, et al. A metaanalysis of cytokines in major depression. Biol Psychiatry. 2010;67(5):446-57.

9. Valkanova V, Ebmeier KP, Allan CL. CRP, IL-6 and depression: a systematic review and meta-analysis of longitudinal studies. J Affect Disord. 2013;150(3):736-44.

10. Dahl J, Ormstad H, Aass HC, Malt UF, Bendz LT, Sandvik L, et al. The plasma levels of various cytokines are increased during ongoing depression and are reduced to normal levels after recovery. Psychoneuroendocrinology. 2014:45:77-86.

11. Sasayama D, Hattori K, Wakabayashi C, Teraishi T, Hori H, Ota M, et al. Increased cerebrospinal fluid interleukin-6 levels in patients with schizophrenia and those with major depressive disorder. J Psychiatr Res. 2013:47:401-6.

12. Lindqvist $D$, Janelidze $S$, Hagell $P$, Erhardt $S$, Samuelsson M, Minthon $L$, et al. Interleukin-6 is elevated in the cerebrospinal fluid of suicide attempters and related to symptom severity. Biol Psychiatry. 2009;66(3):287-92.

13. Dantzer R, O'Connor JC, Lawson MA, Kelley KW. Inflammation-associated depression: from serotonin to kynurenine. Psychoneuroendocrinology. 2011;36(3):426-36. 
14. Erhardt S, Lim CK, Linderholm KR, Janelidze S, Lindqvist D, Samuelsson M, et al. Connecting inflammation with glutamate agonism in suicidality. Neuropsychopharmacology. 2013;38(5):743-52.

15. Bay-Richter C, Linderholm KR, Lim CK, Samuelsson M, Träskman-Bendz L, Guillemin GJ, et al. A role for inflammatory metabolites as modulators of the glutamate $\mathrm{N}$-methyl-D-aspartate receptor in depression and suicidality. Brain Behav Immun. 2015;43:110-7.

16. Myint AM, Kim YK. Cytokine-serotonin interaction through IDO: a neurodegeneration hypothesis of depression. Med Hypotheses. 2003;61(5-6):519-25.

17. Schwarcz R, Bruno JP, Muchowski PJ, Wu HQ. Kynurenines in the mammalian brain: when physiology meets pathology. Nat Rev Neurosci. 2003;13(7):465-77.

18. Guillemin GJ. Quinolinic acid, the inescapable neurotoxin. FEBS J. 2012;279(8):1356-65

19. Kim H, Chen L, Lim G, Sung B, Wang S, McCabe MF, et al. Brain indoleamine 2,3-dioxygenase contributes to the comorbidity of pain and depression. J Clin Invest. 2012;122(8):2940-54.

20. Schwieler L, Larsson MK, Skogh E, Kegel ME, Orhan F, Abdelmoaty S, et al. Increased levels of IL-6 in the cerebrospinal fluid of patients with chronic schizophrenia-significance for activation of the kynurenine pathway. J Psychiatry Neurosci. 2015;40(2):126-33.

21. Urata $Y$, Koga $K$, Hirota $Y$, Akiyama I, Izumi G, Takamura $M$, et al. IL-1ß increases expression of tryptophan 2,3-dioxygenase and stimulates tryptophan catabolism in endometrioma stromal cells. Am J Reprod Immunol. 2014;72(5):496-503.

22. Erhardt S, Blennow K, Nordin C, Skogh E, Lindström LH, Engberg G. Kynurenic acid levels are elevated in the cerebrospinal fluid of patients with schizophrenia. Neurosci Lett. 2001;313(1-2):96-8.

23. Schwarcz R, Rassoulpour A, Wu HQ, Medoff D, Tamminga CA, Roberts RC. Increased cortical kynurenate content in schizophrenia. Biol Psychiatry. 2001;50(7):521-30.

24. Nilsson LK, Linderholm KR, Engberg G. Elevated levels of kynurenic acid in the cerebrospinal fluid of male patients with schizophrenia. Schizophr Res. 2005;80(2-3):315-22.

25. Miller $\mathrm{CL}$, Llenos IC, Dulay JR. Upregulation of the initiating step of the kynurenine pathway in postmortem anterior cingulate cortex from individuals with schizophrenia and bipolar disorder. Brain Res. 2006; 16(1073-1074):25-37.

26. Olsson SK, Samuelsson M, Saetre $P$, Lindström L, Jönsson EG, Nordin C, et al Elevated levels of kynurenic acid in the cerebrospinal fluid of patients with bipolar disorder. J Psychiatry Neurosci. 2010;35(3):195-9.

27. Sathyasaikumar KV, Stachowski EK, Wonodi I, Roberts RC, Rassoulpour A, McMahon RP, et al:. Impaired kynurenine pathway metabolism in the prefrontal cortex of individuals with schizophrenia. Schizophr Bull. 2011;37(6):1147-56.

28. Linderholm K, Skogh E, Olsson SK, Dahl ML, Holtze M, Engberg G, et al. Increased levels of kynurenine and kynurenic acid in the CSF of patients with schizophrenia. Schizophr Bul. 2012;38(3):426-32.

29. Steiner J, Walter M, Gos T, Guillemin GJ, Bernstein HG, Sarnyai Z, et al. Severe depression is associated with increased microglial quinolinic acid in subregions of the anterior cingulate gyrus: evidence for an immunemodulated glutamatergic neurotransmission? J Neuroinflammation. 2011;10(8):94

30. Myint AM, Kim YK, Verkerk R, Scharpé S, Steinbusch H, Leonard B. Kynurenine pathway in major depression: evidence of impaired neuroprotection. J Affect Disord. 2007;98(1-2):143-51.

31. Maes M, Galecki $P$, Verkerk R, Rief W. Somatization, but not depression, is characterized by disorders in the tryptophan catabolite (TRYCAT) pathway, indicating increased indoleamine 2,3-dioxygenase and lowered kynurenine aminotransferase activity. Neuro Endocrinol Lett. 2011;32(3):264-73.

32. Savitz J, Drevets WC, Smith CM, Victor TA, Wurfel BE, Bellgowan PS, et al. Putative neuroprotective and neurotoxic kynurenine pathway metabolites are associated with hippocampal and amygdalar volumes in subjects with major depressive disorder. Neuropsychopharmacology. 2015;40(2):463-71.

33. Savitz J, Drevets WC, Wurfel BE, Ford BN, Bellgowan PS, Victor TA, et al. Reduction of kynurenic acid to quinolinic acid ratio in both the depressed and remitted phases of major depressive disorder. Brain Behav Immun. 2015:46:55-9.

34. Rush AJ, Trivedi MH, Wisniewski SR, Nierenberg AA, Stewart JW, Warden D, et al. Acute and longer-term outcomes in depressed outpatients requiring one or several treatment steps: a STAR*D. Report Am J Psychiatry. 2006;163(11):1905-17.
35. Katz MM, Tekell JL, Bowden CL, Brannan S, Houston JP, Berman N, et al. Onset and early behavioral effects of pharmacologically different antidepressants and placebo in depression. Neuropsychopharmacology. 2004:29(3):566-79.

36. Baldinger $\mathrm{P}$, Lotan $\mathrm{A}$, Frey $\mathrm{R}$, Kasper $\mathrm{S}$, Lerer $\mathrm{B}$, Lanzenberger $\mathrm{R}$. Neurotransmitters and electroconvulsive therapy. J ECT. 2014;30(2):116-21.

37. Haskett RF. Electroconvulsive therapy's mechanism of action: neuroendocrine hypotheses. J ECT. 2014;30(2):107-10.

38. Bouckaert F, Sienaert P, Obbels J, Dols A, Vandenbulcke M, Stek M, et al. ECT: its brain enabling effects: a review of electroconvulsive therapyinduced structural brain plasticity. J ECT. 2014;30(2):143-51.

39. Sheehan DV, Lecrubier $Y$, Sheehan $\mathrm{KH}$, Amorim $\mathrm{P}$, Janavs J, Weiller $E$, et al. The Mini-International Neuropsychiatric Interview (M.I.N.I.): the development and validation of a structured diagnostic psychiatric interview for DSM-IV and ICD-10. J Clin Psychiatry. 1998;59(20):22-33.

40. Montgomery SA, Asberg M. A new depression scale designed to be sensitive to change. Br J Psychiatry. 1979;134(4):382-9.

41. First MB, Spitzer RI, Gibbon M, et al. Structured Clinical Interview for DMS-IV Axis I Disorders, Clinician Version (SCID-CV). Washington: American Psychiatric Press, inc; 1996.

42. Young JJ, Bruno D, Pomara N. A review of the relationship between proinflammatory cytokines and major depressive disorder. J Affect Disord. 2014;169(12):15-20.

43. Howren MB, Lamkin DM, Suls J. Associations of depression with C-reactive protein, IL-1, and IL-6: a meta-analysis. Psychosom Med. 2009;71(2):171-86.

44. Theofylaktopoulou D, Midttun $\varnothing$, Ulvik A, Ueland PM, Tell GS, Vollset SE, et al. A community-based study on determinants of circulating markers of cellular immune activation and kynurenines: the Hordaland Health Study. Clin Exp Immunol. 2013;173(1):121-30.

45. Deac OM, Mills JL, Shane B, Midttun $\varnothing$, Ueland PM, Brosnan JT, et al. Tryptophan catabolism and vitamin B-6 status are affected by gender and lifestyle factors in healthy young adults. J Nutr. 2015;145(4):701-7.

46. Claes S, Myint AM, Domschke K, Del-Favero J, Entrich K, Engelborghs S, et al. The kynurenine pathway in major depression: haplotype analysis of three related functional candidate genes. Psychiatry Res. 2011;188(3):355-60.

47. Guloksuz S, Arts B, Walter S, Drukker M, Rodriguez L, Myint AM, et al. The impact of electroconvulsive therapy on the tryptophan-kynurenine metabolic pathway. Brain Behav Immun. 2015;48:48-52.

48. DiazGranados N, Ibrahim LA, Brutsche NE, Ameli R, Henter ID, Luckenbaugh DA, et al. Rapid resolution of suicidal ideation after a single infusion of an $\mathrm{N}$-methyl-D-aspartate antagonist in patients with treatment-resistant major depressive disorder. J Clin Psychiatry. 2010;71(12):1605-11.

49. Zarate CA, Singh JB, Carlson PJ, Brutsche NE, Ameli R, Luckenbaugh DA, et al. A randomized trial of an $\mathrm{N}$-methyl-D-aspartate antagonist in treatment-resistant major depression. Arch Gen Psychiatry. 2013;74(4):257-64.

50. Machado-Vieira R, Salvadore G, Diazgranados N, Zarate Jr CA. Ketamine and the next generation of antidepressants with a rapid onset of action. Pharmacol Ther. 2009:123(2):143-50.

51. Kirkegaard C, Møller SE, Bjørum N. Addition of L-tryptophan to electroconvulsive treatment in endogenous depression. A double-blind study. Acta Psychiatr Scand. 1978;58(5):457-62.

52. Mokhtar AS, Morgan CJ, Bradley DM, Badawy AA. No early effects of electroconvulsive therapy on tryptophan metabolism and disposition in endogenous depression. Biol Psychiatry. 1997;42(3):201-5.

53. Whalley LJ, Yates CM, Christie JE. Effect of electroconvulsive therapy (ECT) on plasma tryptophan. Psychol Med. 1998;10(2):377-80.

54. Palmio J, Huuhka M, Saransaari P, Oja SS, Peltola J, Leinonen E, et al. Changes in plasma amino acids after electroconvulsive therapy of depressed patients. Psychiatry Res. 2005;137(3):183-90.

55. Hoekstra R, van den Broek WW, Fekkes D, Bruijn JA, Mulder PG, Pepplinkhuizen L. Effect of electroconvulsive therapy on biopterin and large neutral amino acids in severe, medication-resistant depression. Psychiatry Res. 2001;103(2-3):115-23.

56. Okuno E, Kido R. Kynureninase and kynurenine 3-hydroxylase in mammalian tissues. Adv Exp Med Biol. 1991;294:167-76.

57. Bender DA, McCreanor GM. Kynurenine hydroxylase: a potential rate-limiting enzyme in tryptophan metabolism. Biochem Soc Trans. 1985;13(2):441-3. 